\title{
TimberTower gewinnt Deutschen Holzbaupreis
}

Im Rahmen der diesjährigen Ligna verlieh der Zentralverband Deutsches Baugewerbe der TimberTower GmbH den Deutschen Holzbaupreis. Die Jury würdigte den unter dem Markennamen TimberTower bekannten und unter Einsatz moderner Klebtechnik gebauten 100 Meter hohen Holzturm für Windkraftanlagen als weltweit einzigartige Konstruktion und auch die Darstellung der Wettbewerbsfähigkeit des Baustoffes Holz im Vergleich zu herkömmlich eingesetzten Materialien. Dazu Geschäftsführer Holger Giebel: „Wir bekamen
Preise, bevor der Turm stand, als wir ihn bauten und jetzt im laufenden Betrieb. Holz ist unschlagbar. Ich danke der Jury für ihre schöne Entscheidung und allen, die diesen und noch höhere Türme möglich machen."Die TimberTower GmbH gewann 2008 noch als Start-Up den „Klimaschutzpreis“, 2009 den „Schweighofer Prize“ und wurde 2013 zum „Erfolg des Jahrzehnts“ ausgezeichnet. TimberTower arbeitet seit 2012 an einem 140 Meter Holzturm, dessen Realisation ebenfalls nur durch den Einsatz der Klebtechnik möglich ist.

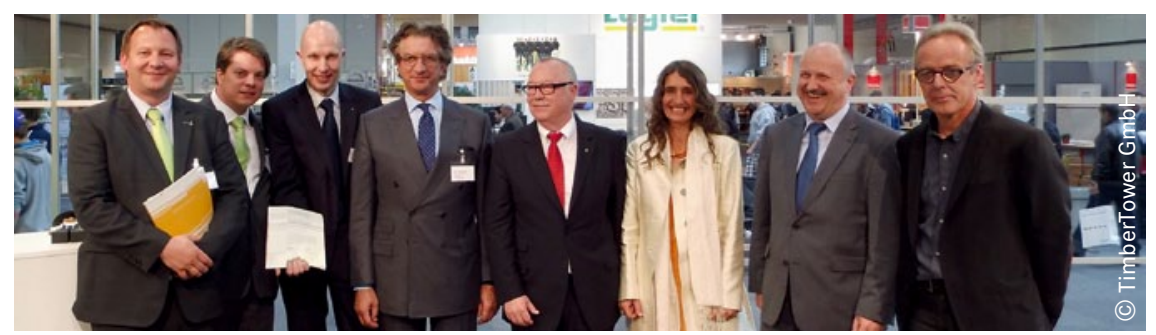

Verleihung des Deutschen Holzbaupreises: (v.l.) Gregor Prass, Geschäftsführer TimberTower, Holger Giebel, Geschäftsführer TimberTower, Ulf Cordes, Geschäftsführer Ing. Holzbau Cordes, Prof. Edwin Kohl, Gesellschafter TimberTower, Ullrich Huth, Holzbau Deutschland (Jury), Sabine Djahanschah, Deutsche Bundestiftung Umwelt (Jury), Vertreter des Bundesministeriums für Verkehr, Bau und Stadtentwicklung, Prof. Hermann Kaufmann, Architekt (Juryvorsitzender)

\section{Butandiol aus nachwachsenden Rohstoffen}

D ie BASF plant die Produktion von 1,4-Butandiol aus nachwachsenden Rohstoffen nach dem patentierten Verfahren des Unternehmens Genomatica, San Diego/Kalifornien. Der einstufige Fermentationsprozess basiert auf Zucker als nachwachsendem Rohstoff.

Die Lizenzvereinbarung erlaubt der BASF den Bau einer World-Scale-Produktionsanlage für BDO aus nachwachsenden Rohstoffen nach dem Genomatica-Verfahren. Außerdem wird Genomatica ihr patentiertes zuckerbasiertes Produktionsverfahren weiterentwi- ckeln, während BASF BDO aus nachwachsenden Rohstoffen herstellt. Muster- und Versuchsmengen für Kunden werden in der zweiten Hälfte des Jahres 2013 zur Verfügung stehen.

BASF produziert derzeit BDO und BDO-Äquivalente an den Standorten in Ludwigshafen/Deutschland, Geismar/Louisiana, Chiba/Japan, Kuantan/ Malaysia und Caojing/China mit einer jährlichen Kapazität von 535.000 Tonnen. BASF hat vor kurzem angekündigt, einen BDO-Komplex in China mit einer Kapazität von 100.000 Jahrestonnen bauen zu wollen.
Bondexpo

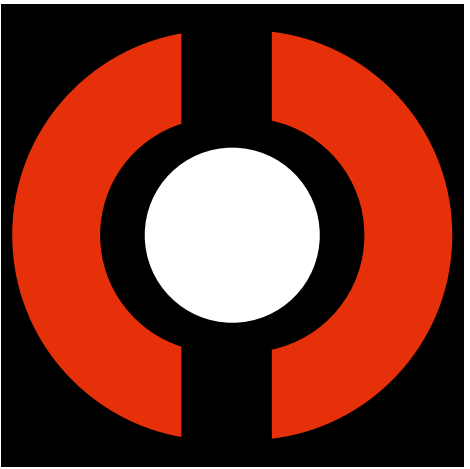

www.bondexpo-messe.de

\section{Bondexpo} Internationale

\section{Fachmesse für Klebtechnologie}

\author{
- Rohstoffe für Kleb- und Dichtstoffe \\ - Maschinen, Anlagen und Zubehör \\ für die Klebstoffherstellende Industrie \\ - Kleb- und Dichtstoffe \\ - Maschinen, Anlagen und Zubehör \\ für die Klebstoffverarbeitende Industrie \\ n Prüf- und Messtechnik
}

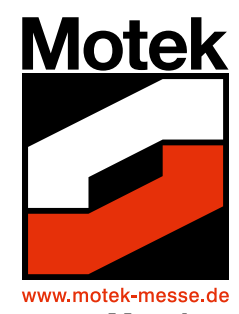

32. Motek

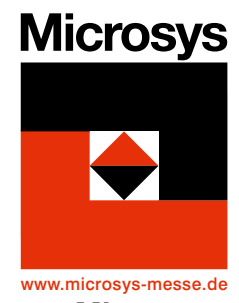

6. Microsys

\section{7. -10.10 .2013$ Stuttgart}

SCHALL MESSEN FÜR MÄRKTE.
WWW.schall-messen.de 